Bull. Austral. Math. Soc.

$47 \mathrm{H} 10,65 \mathrm{~J} 15$

VOL. 55 (1997) [113-130]

\title{
A FAMILY OF CHEBYSHEV-HALLEY TYPE METHODS IN BANACH SPACES
}

\section{J.M. Gutiérrez and M.A. Hernández}

A family of third-order iterative processes (that includes Chebyshev and Halley's methods) is studied in Banach spaces. Results on convergence and uniqueness of solution are given, as well as error estimates. This study allows us to compare the most famous third-order iterative processes.

\section{INTRODUCTION}

Let $X, Y$ be Banach spaces and $F: \Omega \subseteq X \rightarrow Y$ be a nonlinear twice Fréchet differentiable operator in an open convex domain $\Omega_{0} \subseteq \Omega$. Let us assume that $F^{\prime}\left(x_{0}\right)^{-1} \in \mathcal{L}(Y, X)$ exists at some $x_{0} \in \Omega_{0}$, where $\mathcal{L}(Y, X)$ is the set of bounded linear operators from $Y$ into $X$.

Among the third-order methods for solving the equation

$$
F(x)=0
$$

we have:

- Chebyshev's method $[\mathbf{3}, \mathbf{5}, \mathbf{1 5}]$,

$$
x_{n+1}=x_{n}-\left[I+\frac{1}{2} L_{F}\left(x_{n}\right)\right] F^{\prime}\left(x_{n}\right)^{-1} F\left(x_{n}\right), \quad n \geqslant 0,
$$

- Halley's method (or method of tangent hyperbolas) $[1,2,4,6,7,10,13,15$, 20],

$$
x_{n+1}=x_{n}-\left[I+\frac{1}{2} L_{F}\left(x_{n}\right)\left[I-\frac{1}{2} L_{F}\left(x_{n}\right)\right]^{-1}\right] F^{\prime}\left(x_{n}\right)^{-1} F\left(x_{n}\right), \quad n \geqslant 0,
$$

and

- convex acceleration of Newton's method (or super-Halley's method) $[8,12,14]$,

$$
x_{n+1}=x_{n}-\left[I+\frac{1}{2} L_{F}\left(x_{n}\right)\left[I-L_{F}\left(x_{n}\right)\right]^{-1}\right] F^{\prime}\left(x_{n}\right)^{-1} F\left(x_{n}\right), \quad n \geqslant 0 .
$$

Received 26th February, 1996

Supported in part by a grant of the University of La Rioja.

Copyright Clearance Centre, Inc. Serial-fee code: 0004-9729/97 $\$$ A2.00+0.00. 
We have denoted by $I$ the identity operator on $X$ and by $L_{F}(x)$ the linear operator defined by

$$
L_{F}(x)=F^{\prime}(x)^{-1} F^{\prime \prime}(x) F^{\prime}(x)^{-1} F(x), \quad x \in X,
$$

provided than $F^{\prime}(x)^{-1}$ exists. This operator and its connection with Newton's method were studied in [11]. For a real function $f$, the expression

$$
L_{f}(t)=\frac{f(t) f^{\prime \prime}(t)}{f^{\prime}(t)^{2}}
$$

is a measure of the convexity of $f$ at $t$, the degree of logarithmic convexity [13].

In view of these methods, we define for $\alpha \in[0,1], x_{\alpha, 0}=x_{0} \in \Omega_{0}$ and $n \geqslant 0$ the following one-parameter family of iterative processes

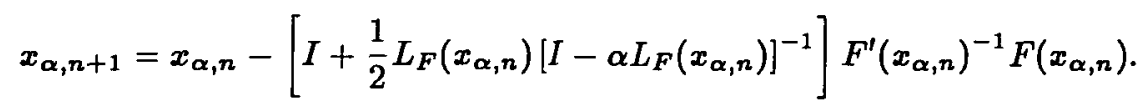

This family extends the family of scalar iterative processes considered by Hernández and Salanova in [15], and includes, as particular cases, Chebyshev's method $(\alpha=0)$, Halley's method $(\alpha=1 / 2)$ and convex acceleration of Newton's method $(\alpha=1)$.

In this paper, we obtain results on existence and uniqueness of solution of (1), convergence of the sequences (2) to this solution under Kantorovich-type assumptions (see $[16,17,18])$ and error estimates. Finally, we give some examples to illustrate the previous results, analysing the velocity of convergence of different methods and comparing our error bounds with those that have been given by other authors.

\section{Preliminaries}

Following Yamamoto [20], we assume throughout this paper that

(i) There exists a continous linear operator $\Gamma_{0}=F^{\prime}\left(x_{0}\right)^{-1}, x_{0} \in \Omega_{0}$.

(ii) $\left\|\Gamma_{0}\left(F^{\prime \prime}(x)-F^{\prime \prime}(y)\right)\right\| \leqslant k\|x-y\|, x, y \in \Omega_{0}, k \geqslant 0$.

(iii) $\left\|\Gamma_{0} F\left(x_{0}\right)\right\| \leqslant a, \quad\left\|\Gamma_{0} F^{\prime \prime}\left(x_{0}\right)\right\| \leqslant b$.

(iv) The equation

$$
p(t) \equiv \frac{k}{6} t^{3}+\frac{b}{2} t^{2}-t+a=0
$$

has one negative root and two positive roots $r_{1}$ and $r_{2}\left(r_{1} \leqslant r_{2}\right)$ if $k>0$, or has two positive roots $r_{1}$ and $r_{2}\left(r_{1} \leqslant r_{2}\right)$ if $k=0$. Equivalently,

$$
a \leqslant \frac{b^{2}+4 k-b \sqrt{b^{2}+2 k}}{3 k\left(b+\sqrt{b^{2}+2 k}\right)} \text { if } k>0,
$$

or $a b \leqslant 1 / 2$ if $k=0$. 
For each $\alpha \in[0,1]$, let us define the scalar sequence $\left\{t_{\alpha, n}\right\}$ by

$$
t_{\alpha, 0}=t_{0}=0, t_{\alpha, n+1}=t_{\alpha, n}-\left[1+\frac{L_{p}\left(t_{\alpha, n}\right)}{2\left(1-\alpha L_{p}\left(t_{\alpha, n}\right)\right)}\right] \frac{p\left(t_{\alpha, n}\right)}{p^{\prime}\left(t_{\alpha, n}\right)}, n \geqslant 0,
$$

where $p$ is the polynomial defined by (3).

We write $\Gamma_{\alpha, n}=F^{\prime}\left(x_{\alpha, n}\right)^{-1}$ and $H_{\alpha, n}=\left[I-\alpha L_{F}\left(x_{\alpha, n}\right)\right]^{-1}$, when they exist.

Under the assumptions (i)-(iv) we prove that the sequences $\left\{t_{\alpha, n}\right\}$ and $\left\{x_{\alpha, n}\right\}$, are well-defined, converge to $r_{1}$ and a solution $x^{*}$ of (1) respectively, and

$$
\begin{gathered}
\left\|x_{\alpha, n+1}-x_{\alpha, n}\right\| \leqslant t_{\alpha, n+1}-t_{\alpha, n}, \quad n \geqslant 0, \\
\left\|x^{*}-x_{\alpha, n}\right\| \leqslant r_{1}-t_{\alpha, n}, \quad n \geqslant 0 .
\end{gathered}
$$

That is, $\left\{t_{\alpha, n}\right\}$ is a majorising sequence of $\left\{x_{\alpha, n}\right\}$ (see $[16,19]$ ).

First, we give a general result on convergence of scalar sequences that includes, as a particular case, the family $\left\{t_{\alpha, n}\right\}$ defined by (4).

LEMma 2.1. Let $p$ be the polynomial defined in (3) with two positive roots $r_{1} \leqslant r_{2}$. Then the sequences

$$
s_{\alpha, 0}=0, s_{\alpha, n+1}=G_{\alpha}\left(s_{\alpha, n}\right), n \geqslant 0,
$$

where

$$
G_{\alpha}(s)=s-\left[1+\frac{L_{p}(s)}{2\left(1-\alpha L_{p}(s)\right)}\right] \frac{p(s)}{p^{\prime}(s)}
$$

and

$$
\alpha \leqslant \min \left\{2,1-\frac{L_{p^{\prime}}\left(r_{1}\right)}{3}\right\},
$$

converge to $r_{1}$. Moreover these sequences are increasing to $r_{1}$.

Proof: Under the previous assumptions for $p$, it is well known, [2], that

$$
0 \leqslant L_{p}(t)<\frac{1}{2}, \quad \text { for } t \in\left[0, r_{1}\right]
$$

Therefore we have $s_{\alpha, n+1} \geqslant s_{\alpha, n}$.

On the other hand, we can write

$$
G_{\alpha}^{\prime}(s)=\frac{L_{p}(s)^{2}}{2\left(1-\alpha L_{p}(s)\right)^{2}}\left[3(1-\alpha)+\alpha(2 \alpha-1) L_{p}(s)-L_{p^{\prime}}(s)\right] .
$$

Taking into account that $L_{p^{\prime}}$ is negative and increasing for $s \in\left[0, r_{1}\right]$, we have that $G_{\alpha}^{\prime}(s) \geqslant 0, s \in\left[0, r_{1}\right]$ and the result follows.

Besides, the convergence of the sequences $\left\{t_{\alpha, n}\right\}$ is of third order. That follows as a consequence of the following result of Gander $[\mathbf{1 0}]$. 
LEMMA 2.2. Let $r_{1}$ be a simple zero of $p$ and $\Delta$ any function satisfying $\Delta(0)=$ $1, \Delta^{\prime}(0)=1 / 2,\left|\Delta^{\prime \prime}(0)\right|<\infty$. The iteration

is of third order.

$$
t_{n+1}=t_{n}-\Delta\left(L_{p}\left(t_{n}\right)\right) \frac{p\left(t_{n}\right)}{p^{\prime}\left(t_{n}\right)}
$$

Although the convergence is cubic in general, in [12] it was established that the sequence $\left\{t_{1, n}\right\}$ (called convex acceleration of Newton's method) has convergence of order four when $p$ is a quadratic polynomial. Moreover, in this case, two iterations of Newton's method are equal to one iteration of the sequence $\left\{t_{1, n}\right\}$.

Now, we center our study on obtaining error expressions for the sequences (4). When $p$ is a quadratic polynomial, following Ostrowski [17], we derive the following error bounds.

LEMmA 2.3. Let $p$ be the polynomial given by (3) with $k=0$, that is

$$
p(t)=\frac{b}{2} t^{2}-t+a
$$

We assume that $p$ has two positive roots $r_{1} \leqslant r_{2}$. Let $\left\{t_{\alpha, n}\right\}$ be the sequence defined in (4).

(a) When $r_{1}<r_{2}$, put $\theta=\frac{r_{1}}{r_{2}}$ and $R_{\alpha}=\frac{\theta+2(1-\alpha)}{1+2(1-\alpha) \theta}$. Then we have:

$$
\begin{aligned}
& \left(\mathrm{a}_{1}\right) \text { If } \alpha \in[0,1 / 2) \text { and } a b<\frac{\sqrt{8(1-\alpha)}}{(1+\sqrt{2(1-\alpha)})^{2}} \text {, } \\
& \left(r_{2}-r_{1}\right) \frac{\theta^{3^{n}}}{1-\theta^{3^{n}}} \leqslant\left(r_{2}-r_{1}\right) \frac{\left[\sqrt{R_{\alpha}} \theta\right]^{3^{n}}}{\sqrt{R_{\alpha}}-\left[\sqrt{R_{\alpha}} \theta\right]^{3^{n}}} \leqslant r_{1}-t_{\alpha, n} \\
& \leqslant\left(r_{2}-r_{1}\right) \frac{[\sqrt{2(1-\alpha)} \theta]^{3^{n}}}{\sqrt{2(1-\alpha)}-[\sqrt{2(1-\alpha)} \theta]^{3^{n}}} \text {. }
\end{aligned}
$$

(a $\mathbf{a}_{2}$ If $\alpha=1 / 2$,

$$
\begin{gathered}
r_{1}-t_{1 / 2, n}=\left(r_{2}-r_{1}\right) \frac{\theta^{3^{n}}}{1-\theta^{3^{n}}} . \\
\left(a_{3}\right) \text { If } \alpha \in(1 / 2,1), \\
\left(r_{2}-r_{1}\right) \frac{[\sqrt{2(1-\alpha)} \theta]^{3^{n}}}{\sqrt{2(1-\alpha)}-[\sqrt{2(1-\alpha)} \theta]^{3^{n}}} \leqslant r_{1}-t_{\alpha, n} \\
\leqslant\left(r_{2}-r_{1}\right) \frac{\left[\sqrt{R_{\alpha}} \theta\right]^{3^{n}}}{\sqrt{R_{\alpha}}-\left[\sqrt{R_{\alpha}} \theta\right]^{3^{n}}} \leqslant\left(r_{2}-r_{1}\right) \frac{\theta^{3^{n}}}{1-\theta^{3^{n}}}
\end{gathered}
$$


(a) Finally, if $\alpha=1$,

$$
r_{1}-t_{1, n}=\frac{\left(r_{2}-r_{1}\right) \theta^{4^{n}}}{1-\theta^{4^{n}}}
$$

(b) When $r_{1}=r_{2}$, we have

$$
r_{1}-t_{\alpha, n}=r_{1}\left(\frac{3-2 \alpha}{4(2-\alpha)}\right)^{n}
$$

ProOF: Let us write $a_{\alpha, n}=r_{1}-t_{\alpha, n}, b_{\alpha, n}=r_{2}-t_{\alpha, n}, n \geqslant 0$. Thus

$$
p\left(t_{\alpha, n}\right)=\frac{b}{2} a_{\alpha, n} b_{\alpha, n}, \quad p^{\prime}\left(t_{\alpha, n}\right)=-\frac{b}{2}\left(a_{\alpha, n}+b_{\alpha, n}\right) .
$$

By (4) we have

(6) $\quad a_{\alpha, n+1}=r_{1}-t_{\alpha, n+1}=a_{\alpha, n}^{3} \frac{a_{\alpha, n}+2(1-\alpha) b_{\alpha, n}}{\left(a_{\alpha, n}+b_{\alpha, n}\right)\left(a_{\alpha, n}^{2}+b_{\alpha, n}^{2}+2(1-\alpha) a_{\alpha, n} b_{\alpha, n}\right)}$, and similarly

$$
b_{\alpha, n+1}=r_{2}-t_{\alpha, n+1}=b_{\alpha, n}^{3} \frac{b_{\alpha, n}+2(1-\alpha) a_{\alpha, n}}{\left(a_{\alpha, n}+b_{\alpha, n}\right)\left(a_{\alpha, n}^{2}+b_{\alpha, n}^{2}+2(1-\alpha) a_{\alpha, n} b_{\alpha, n}\right)} .
$$

If $r_{1}<r_{2}$, we put $\theta=r_{1} / r_{2}<1$ and $\mu_{\alpha, n}=\frac{a_{\alpha, n}}{b_{\alpha, n}}$ to obtain

$$
\mu_{\alpha, n+1}=\mu_{\alpha, n}^{3} \frac{\mu_{\alpha, n}+2(1-\alpha)}{1+2(1-\alpha) \mu_{\alpha, n}}
$$

Taking into account that the function

$$
\frac{x+2(1-\alpha)}{1+2(1-\alpha) x}
$$

is decreasing when $\alpha \in[0,1 / 2)$, is constant when $\alpha=1 / 2$, is increasing when $\alpha \in$ $(1 / 2,1)$ and is the identity when $\alpha=1$, we obtain the first part.

If $r_{1}=r_{2}$, then $a_{\alpha, n}=b_{\alpha, n}$. Therefore, from (6), we have

$$
a_{\alpha, n+1}=a_{\alpha, n} \frac{3-2 \alpha}{4(2-\alpha)}
$$

By recurrence, the second part holds.

When $k>0$ the real sequences $\left\{t_{\alpha, n}\right\}$ in (4) are obtained from a cubic polynomial. In this case, it is difficult to obtain error bounds following Ostrowski's method. In the next lemma, we establish estimates for the error in this situation by using a new procedure. 
LEMMA 2.4. Let $p$ be the polynomial given by (3) with $k>0$, that is

$$
p(t)=\frac{k}{6} t^{3}+\frac{b}{2} t^{2}-t+a
$$

Let us assume that $p$ has two positive roots $r_{1} \leqslant r_{2}$ and a negative root, $-r_{0}$. Let $\left\{t_{\alpha, n}\right\}$ be the sequence defined in (4). Then, if $r_{1}<r_{2}$ and $\theta=\sqrt{\lambda_{\alpha}} r_{1} / r_{2}<1$,

$$
r_{1}-t_{n} \sim \frac{\left(r_{2}-r_{1}\right) \theta^{3^{n}}}{\sqrt{\lambda_{\alpha}}-\theta^{3^{n}}}, \quad n \geqslant 0
$$

where

$$
\lambda_{\alpha}=\frac{\left(r_{2}-r_{1}\right)\left(r_{0}+r_{1}\right)+2(1-\alpha)\left(r_{0}+2 r_{1}-r_{2}\right)^{2}}{\left(r_{0}+r_{1}\right)^{2}}<1, \quad \theta=\sqrt{\lambda_{\alpha}} \frac{r_{1}}{r_{2}}<1 .
$$

If $r_{1}=r_{2}$, we have

$$
r_{1}-t_{n} \sim r_{1}\left(\frac{3-2 \alpha}{4(2-\alpha)}\right)^{n}
$$

Proof: The polynomial $p$ defined above can be written in the form

$$
p(t)=\frac{k}{6}\left(r_{1}-t\right)\left(r_{2}-t\right)\left(r_{0}+t\right)
$$

Let us write $a_{\alpha, n}=r_{1}-t_{\alpha, n}, b_{\alpha, n}=r_{2}-t_{\alpha, n}$ and

$$
Q\left(t_{\alpha, n}\right)=\frac{b_{\alpha, n}^{3} a_{\alpha, n+1}}{a_{\alpha, n}^{3} b_{\alpha, n+1}}=\frac{\left(r_{1}-G_{\alpha}\left(t_{\alpha, n}\right)\right)\left(r_{2}-t_{\alpha, n}\right)^{3}}{\left(r_{2}-G_{\alpha}\left(t_{\alpha, n}\right)\right)\left(r_{1}-t_{\alpha, n}\right)^{3}}
$$

with $G_{\alpha}$ defined by (5).

As $G_{\alpha}\left(r_{1}\right)=r_{1}, G_{\alpha}^{\prime}\left(r_{1}\right)=G_{\alpha}^{\prime \prime}\left(r_{1}\right)=0$, we have for $t$ close to $r_{1}$

$$
\begin{aligned}
Q(t) & \sim\left(r_{2}-r_{1}\right)^{2} \lim _{t \rightarrow r_{1}} \frac{r_{1}-G_{\alpha}(t)}{\left(r_{1}-t\right)^{3}}=\frac{G_{\alpha}^{\prime \prime \prime}\left(r_{1}\right)}{6}\left(r_{2}-r_{1}\right)^{2} \\
& =\frac{3(1-\alpha) p^{\prime \prime}\left(r_{1}\right)^{2}-p^{\prime \prime \prime}\left(r_{1}\right) p^{\prime}\left(r_{1}\right)}{6 p^{\prime}\left(r_{1}\right)^{2}}\left(r_{2}-r_{1}\right)^{2} \\
& =\frac{\left(r_{2}-r_{1}\right)\left(r_{0}+r_{1}\right)+2(1-\alpha)\left(r_{0}+2 r_{1}-r_{2}\right)^{2}}{\left(r_{0}+r_{1}\right)^{2}}=\lambda_{\alpha} .
\end{aligned}
$$

Since $t_{\alpha, n} \rightarrow r_{1}$ when $n \rightarrow \infty$, we obtain

$$
\frac{a_{\alpha, n}}{b_{\alpha, n}} \sim\left(\frac{a_{\alpha, n-1}}{b_{\alpha, n-1}}\right)^{3} \lambda_{\alpha} \sim \cdots \sim\left(\sqrt{\lambda_{\alpha}} \frac{r_{1}}{r_{2}}\right)^{3^{n}} \frac{1}{\sqrt{\lambda_{\alpha}}}
$$


and the first part holds.

If $r_{1}=r_{2}$, let

$$
\widetilde{Q}\left(t_{\alpha, n}\right)=\frac{a_{\alpha, n+1}}{a_{\alpha, n}}=\frac{r_{1}-G_{\alpha}\left(t_{\alpha, n}\right)}{r_{1}-t_{\alpha, n}} .
$$

Notice that for $t$ close to $r_{1}$

$$
\widetilde{Q}(t) \sim \widetilde{Q}\left(r_{1}\right)=\frac{3-2 \alpha}{4(2-\alpha)} .
$$

By recurrence, the second part also follows.

We center now our study on the sequences $\left\{x_{\alpha, n}\right\}, \alpha \in[0,1]$, defined in Banach spaces.

LEMMA 2.5. With the above notation and assumptions, we can write $F\left(x_{\alpha, n+1}\right)$ in the following way:

$$
\begin{array}{r}
F\left(x_{\alpha, n+1}\right)=\frac{1}{8} F^{\prime \prime}\left(x_{\alpha, n}\right) y_{\alpha, n}^{2}+\frac{1-\alpha}{2} F^{\prime \prime}\left(x_{\alpha, n}\right) \Gamma_{\alpha, n} F\left(x_{\alpha, n}\right) y_{\alpha, n} \\
+\int_{x_{\alpha, n}}^{x_{\alpha, n+1}}\left[F^{\prime \prime}(x)-F^{\prime \prime}\left(x_{\alpha, n}\right)\right]\left(x_{\alpha, n+1}-x\right) d x
\end{array}
$$

where

$$
y_{\alpha, n}=L_{F}\left(x_{\alpha, n}\right) H_{\alpha, n} \Gamma_{\alpha, n} F\left(x_{\alpha, n}\right)
$$

Proof: By Taylor's formula, and using (2), we deduce

$$
\begin{aligned}
F\left(x_{\alpha, n+1}\right)= & F\left(x_{\alpha, n}\right)+F^{\prime}\left(x_{\alpha, n}\right)\left(x_{\alpha, n+1}-x_{\alpha, n}\right)+\frac{1}{2} F^{\prime \prime}\left(x_{\alpha, n}\right)\left(x_{\alpha, n+1}-x_{\alpha, n}\right)^{2} \\
& +\int_{x_{\alpha, n}}^{x_{\alpha, n+1}}\left[F^{\prime \prime}(x)-F^{\prime \prime}\left(x_{\alpha, n}\right)\right]\left(x_{\alpha, n+1}-x\right) d x \\
=- & \frac{1}{2} F^{\prime \prime}\left(x_{\alpha, n}\right) \Gamma_{\alpha, n} F\left(x_{\alpha, n}\right) H_{\alpha, n} \Gamma_{\alpha, n} F\left(x_{\alpha, n}\right)+\frac{1}{2} F^{\prime \prime}\left(x_{\alpha, n}\right)\left(\Gamma_{\alpha, n} F\left(x_{\alpha, n}\right)\right)^{2} \\
& +\frac{1}{8} F^{\prime \prime}\left(x_{\alpha, n}\right) y_{\alpha, n}^{2}+\frac{1}{2} F^{\prime \prime}\left(x_{\alpha, n}\right) \Gamma_{\alpha, n} F\left(x_{\alpha, n}\right) y_{\alpha, n} \\
& +\int_{x_{\alpha, n}}^{x_{\alpha, n+1}}\left[F^{\prime \prime}(x)-F^{\prime \prime}\left(x_{\alpha, n}\right)\right]\left(x_{\alpha, n+1}-x\right) d x .
\end{aligned}
$$

As $H_{\alpha, n}=I+\alpha L_{F}\left(x_{\alpha, n}\right) H_{\alpha, n}$, the result holds.

LEMMA 2.6. The iterates (2) are well defined for $\alpha \in[0,1]$ and $n \geqslant 0$, converge to $x^{*}$, a solution of (1), and

$$
\begin{gathered}
\left\|x_{\alpha, n+1}-x_{\alpha, n}\right\| \leqslant t_{\alpha, n+1}-t_{\alpha, n} \\
\left\|x^{*}-x_{\alpha, n}\right\| \leqslant r_{1}-t_{\alpha, n}
\end{gathered}
$$


Proof: For each $\alpha \in[0,1]$ we prove for $n \geqslant 0$ :

$$
\begin{aligned}
& {\left[\mathrm{I}_{n}\right] } \Gamma_{\alpha, n}=F^{\prime}\left(x_{\alpha, n}\right)^{-1} \text { exists }\left(\Gamma_{\alpha, 0}=\Gamma_{0}=F^{\prime}\left(x_{0}\right)^{-1}\right) . \\
& {\left[\mathrm{II}_{n}\right] }\left\|\Gamma_{0} F^{\prime \prime}\left(x_{\alpha, n}\right)\right\| \leqslant-\frac{p^{\prime \prime}\left(t_{\alpha, n}\right)}{p^{\prime}\left(t_{0}\right)} . \\
& {\left[\mathrm{III}_{n}\right] }\left\|\Gamma_{\alpha, n} F^{\prime}\left(x_{0}\right)\right\| \leqslant \frac{p^{\prime}\left(t_{0}\right)}{p^{\prime}\left(t_{\alpha, n}\right)} . \\
& {\left[\mathrm{IV}_{n}\right] }\left\|\Gamma_{0} F\left(x_{\alpha, n}\right)\right\| \leqslant-\frac{p\left(t_{\alpha, n}\right)}{p^{\prime}\left(t_{0}\right)} . \\
& {\left[\mathrm{V}_{n}\right] \quad H_{\alpha, n}=\left[I-\alpha L_{F}\left(x_{\alpha, n}\right)\right]^{-1} \text { exists and }\left\|H_{\alpha, n}\right\| \leqslant \frac{1}{1-\alpha L_{p}\left(t_{\alpha, n}\right)} . }
\end{aligned}
$$

Since $p$ defined in $(3)$ satisfies $L_{p}(t) \leqslant 1 / 2$, (see [2]), then $\left[V_{n+1}\right]$ follows as a consequence of $\left[\mathrm{II}_{n+1}\right],\left[\mathrm{III}_{n+1}\right]$ and $\left[\mathrm{IV}_{n+1}\right]$. Thus, we prove $\left[\mathrm{I}_{n+1}\right]-\left[\mathrm{IV}_{n+1}\right]$ using induction. Applying Altman's technique, (see [2] or [20]), $\left[\mathrm{I}_{n+1}\right],\left[\mathrm{II}_{n+1}\right]$ and $\left[\mathrm{III}_{n+1}\right]$ follow immediately.

To prove $\left[\mathrm{IV}_{n+1}\right]$, let us write $\nu_{\alpha, n}=L_{p}\left(t_{\alpha, n}\right)$. Then

$$
\left\|y_{\alpha, n}\right\| \leqslant\left\|L_{F}\left(x_{\alpha, n}\right)\right\|\left\|H_{\alpha, n}\right\|\left\|\Gamma_{\alpha, n} F\left(x_{\alpha, n}\right)\right\| \leqslant-\frac{\nu_{\alpha, n} p\left(t_{\alpha, n}\right)}{\left(1-\alpha \nu_{\alpha, n}\right) p^{\prime}\left(t_{\alpha, n}\right)},
$$

were $y_{\alpha, n}$ is given by (8). Therefore, from Lemma 2.5,

$$
\left\|\Gamma_{0} F\left(x_{\alpha, n+1}\right)\right\| \leqslant \frac{1}{8} \frac{\nu_{\alpha, n}^{3} p\left(t_{\alpha, n}\right)}{\left(1-\alpha \nu_{\alpha, n}\right)^{2}}+\frac{1-\alpha}{2} \frac{\nu_{\alpha, n}^{2} p\left(t_{\alpha, n}\right)}{1-\alpha \nu_{\alpha, n}}+\frac{k}{6}\left(t_{\alpha, n+1}-t_{\alpha, n}\right)^{3}
$$

Repeating the same process for the polynomial $p$, we obtain

$$
p\left(t_{\alpha, n+1}\right)=\frac{1}{8} \frac{\nu_{\alpha, n}^{3} p\left(t_{\alpha, n}\right)}{\left(1-\alpha \nu_{\alpha, n}\right)^{2}}+\frac{1-\alpha}{2} \frac{\nu_{\alpha, n}^{2} p\left(t_{\alpha, n}\right)}{1-\alpha \nu_{\alpha, n}}+\frac{k}{6}\left(t_{\alpha, n+1}-t_{\alpha, n}\right)^{3},
$$

and consequently,

$$
\left\|\Gamma_{0} F\left(x_{\alpha, n+1}\right)\right\| \leqslant p\left(t_{\alpha, n+1}\right)=-\frac{p\left(t_{\alpha, n+1}\right)}{p^{\prime}\left(t_{0}\right)}
$$

So we conclude the induction.

Next, we have

$$
\begin{aligned}
\left\|x_{\alpha, n+1}-x_{\alpha, n}\right\| & =\left\|\left[I+\frac{1}{2} L_{F}\left(x_{\alpha, n}\right) H_{\alpha, n}\right] \Gamma_{\alpha, n} F\left(x_{\alpha, n}\right)\right\| \\
& \leqslant\left[1+\frac{L_{p}\left(t_{\alpha, n}\right)}{2\left(1-\alpha L_{p}\left(t_{\alpha, n}\right)\right)}\right] \frac{p\left(t_{\alpha, n}\right)}{p^{\prime}\left(t_{\alpha, n}\right)}=t_{\alpha, n+1}-t_{\alpha, n},
\end{aligned}
$$


then (9) holds, and $\left\{t_{\alpha, n}\right\}$ majorises $\left\{x_{\alpha, n}\right\}$. The convergence of $\left\{t_{\alpha, n}\right\}$ (see Lemma 2.1 and its note) implies the convergence of $\left\{x_{\alpha, n}\right\}$ to a limit $x^{*}$. By letting $n \rightarrow \infty$ in (11), we deduce $F\left(x^{*}\right)=0$.

Finally, for $p \geqslant 0$,

$$
\left\|x_{\alpha, n+p}-x_{\alpha, n}\right\| \leqslant t_{\alpha, n+p}-t_{\alpha, n}
$$

and by letting $p \rightarrow \infty$ we obtain (10).

LеммA 2.7. Under the previous assumptions we have, for $0 \leqslant \alpha \leqslant 1 / 2$

$$
\left\|x^{*}-x_{\alpha, n+1}\right\| \leqslant\left(r_{1}-t_{\alpha, n+1}\right)\left(\frac{\left\|x^{*}-x_{\alpha, n}\right\|}{r_{1}-t_{\alpha, n}}\right)^{2},
$$

and for $1 / 2 \leqslant \alpha \leqslant 1$,

$$
\left\|x^{*}-x_{\alpha, n+1}\right\| \leqslant\left(r_{1}-t_{\alpha, n+1}\right)\left(\frac{\left\|x^{*}-x_{\alpha, n}\right\|}{r_{1}-t_{\alpha, n}}\right)^{3} .
$$

Proof: The argument of Yamamoto (see [12] or [20] for details), and using

$$
I+\frac{1}{2} L_{F}\left(x_{\alpha, n}\right) H_{\alpha, n}=H_{\alpha, n}\left(I-\left(\alpha-\frac{1}{2}\right) L_{F}\left(x_{\alpha, n}\right)\right) \text {, }
$$

shows that

$$
\begin{aligned}
x^{*}-x_{\alpha, n+1}= & -H_{\alpha, n} \Gamma_{\alpha, n} \int_{x_{\alpha, n}}^{x^{*}}\left[F^{\prime \prime}(x)-F^{\prime \prime}\left(x_{\alpha, n}\right)\right]\left(x^{*}-x\right) d x \\
& +\left[I-H_{\alpha, n}\right]\left(x^{*}-x_{\alpha, n}\right)-\frac{1}{2} H_{\alpha, n} \Gamma_{\alpha, n} F^{\prime \prime}\left(x_{\alpha, n}\right)\left(x^{*}-x_{\alpha, n}\right)^{2} \\
& +\frac{1}{2} H_{\alpha, n} L_{F}\left(x_{\alpha, n}\right) \Gamma_{\alpha, n} F\left(x_{\alpha, n}\right)
\end{aligned}
$$

Since $I-H_{\alpha, n}=-\alpha H_{\alpha, n} L_{F}\left(x_{\alpha, n}\right)$, we obtain

$$
\begin{aligned}
x^{*}-x_{\alpha, n+1}= & -H_{\alpha, n} \Gamma_{\alpha, n} \int_{x_{\alpha, n}}^{x^{*}}\left[F^{\prime \prime}(x)-F^{\prime \prime}\left(x_{\alpha, n}\right)\right]\left(x^{*}-x\right) d x \\
& +\left[I-H_{\alpha, n}\right]\left(x^{*}-x_{\alpha, n}\right)+\alpha H_{\alpha, n} L_{F}\left(x_{\alpha, n}\right) \Gamma_{\alpha, n} \int_{x_{\alpha, n}}^{x^{*}} F^{\prime \prime}(x)\left(x^{*}-x\right) d x \\
& +\frac{1}{2} H_{\alpha, n} \Gamma_{\alpha, n} F^{\prime \prime}\left(x_{\alpha, n}\right)\left[\left(\Gamma_{\alpha, n} F\left(x_{\alpha, n}\right)\right)^{2}-\left(x^{*}-x_{\alpha, n}\right)^{2}\right]
\end{aligned}
$$


Then, for $0 \leqslant \alpha<1 / 2$ it follows that

$$
\begin{aligned}
x^{*}-x_{n+1}= & -H_{\alpha, n} \Gamma_{\alpha, n} \int_{x_{\alpha, n}}^{x^{*}}\left[F^{\prime \prime}(x)-F^{\prime \prime}\left(x_{\alpha, n}\right)\right]\left(x^{*}-x\right) d x \\
& +\left(\alpha-\frac{1}{2}\right) H_{\alpha, n} L_{F}\left(x_{\alpha, n}\right) \Gamma_{\alpha, n} \int_{x_{\alpha, n}}^{x^{*}} F^{\prime \prime}(x)\left(x^{*}-x\right) d x \\
& +\frac{1}{2} H_{\alpha, n} \Gamma_{\alpha, n} F^{\prime \prime}\left(x_{\alpha, n}\right)\left[\Gamma_{\alpha, n} \int_{x_{\alpha, n}}^{x^{*}} F^{\prime \prime}(x)\left(x^{*}-x\right) d x\right]\left(x^{*}-x\right) .
\end{aligned}
$$

For $1 / 2 \leqslant \alpha \leqslant 1$, writing

$$
\frac{1}{2}=\left(\alpha-\frac{1}{2}\right)+(1-\alpha)
$$

in (12), we deduce

$$
\begin{aligned}
x^{*}-x_{n+1}= & -H_{\alpha, n} \Gamma_{\alpha, n} \int_{x_{\alpha, n}}^{x^{*}}\left[F^{\prime \prime}(x)-F^{\prime \prime}\left(x_{\alpha, n}\right)\right]\left(x^{*}-x\right) d x \\
& -\left(\alpha-\frac{1}{2}\right) H_{\alpha, n} \Gamma_{\alpha, n} F^{\prime \prime}\left(x_{\alpha, n}\right)\left[\Gamma_{\alpha, n} \int_{x_{\alpha, n}}^{x^{*}} F^{\prime \prime}(x)\left(x^{*}-x\right) d x\right]^{2} \\
& +(1-\alpha) H_{\alpha, n} \Gamma_{\alpha, n} F^{\prime \prime}\left(x_{\alpha, n}\right)\left[\Gamma_{\alpha, n} \int_{x_{\alpha, n}}^{x^{*}} F^{\prime \prime}(x)\left(x^{*}-x\right) d x\right]\left(x^{*}-x\right)
\end{aligned}
$$

Consequently, for $0 \leqslant \alpha<1 / 2$,

$$
\begin{aligned}
\| x^{*}- & x_{\alpha, n+1} \| \\
\leqslant & {\left[-\frac{k\left(r_{1}-t_{\alpha, n}\right)^{3}}{6\left(1-\alpha L_{p}\left(t_{\alpha, n}\right)\right) p^{\prime}\left(t_{\alpha, n}\right)}\right.} \\
& \left.+\frac{p^{\prime \prime}\left(t_{\alpha, n}\right)\left(r_{1}-t_{\alpha, n}\right)}{2\left(1-\alpha L_{p}\left(t_{\alpha, n}\right)\right) p^{\prime}\left(t_{\alpha, n}\right)^{2}} \int_{t_{\alpha, n}}^{r_{1}} p^{\prime \prime}(z)\left(r_{1}-z\right) d z\right]\left(\frac{\left\|x^{*}-x_{\alpha, n}\right\|}{r_{1}-t_{\alpha, n}}\right)^{3} \\
& +\left(\alpha-\frac{1}{2}\right) \frac{L_{p}\left(t_{\alpha, n}\right)}{\left(1-\alpha L_{p}\left(t_{\alpha, n}\right)\right) p^{\prime}\left(t_{\alpha, n}\right)} \int_{t_{\alpha, n}}^{r_{1}} p^{\prime \prime}(z)\left(r_{1}-z\right) d z\left(\frac{\left\|x^{*}-x_{\alpha, n}\right\|}{r_{1}-t_{\alpha, n}}\right)^{2} \\
\leqslant & {\left[-\frac{k\left(r_{1}-t_{\alpha, n}\right)^{3}}{6\left(1-\alpha L_{p}\left(t_{\alpha, n}\right)\right) p^{\prime}\left(t_{\alpha, n}\right)}+\frac{p^{\prime \prime}\left(t_{\alpha, n}\right)\left(r_{1}-t_{\alpha, n}\right)}{2\left(1-\alpha L_{p}\left(t_{\alpha, n}\right)\right) p^{\prime}\left(t_{\alpha, n}\right)^{2}} \int_{t_{\alpha, n}}^{r_{1}} p^{\prime \prime}(z)\left(r_{1}-z\right) d z\right.} \\
& +\left(\alpha-\frac{1}{2}\right) \frac{L_{p}\left(t_{\alpha, n}\right)}{\left(1-\alpha L_{p}\left(t_{\alpha, n}\right)\right) p^{\prime}\left(t_{\alpha, n}\right)} \int_{t_{\alpha, n}}^{r_{1}} p^{\prime \prime}(z)\left(r_{1}-z\right) d z \\
\leqslant & \left(r_{1}-t_{\alpha, n+1}\right)\left(\frac{\left\|x^{*}-x_{\alpha, n}\right\|}{r_{1}-t_{\alpha, n}}\right)^{2} \\
r_{1}-t_{\alpha, n} & \cdot
\end{aligned}
$$


In a similar way, we obtain for $1 / 2 \leqslant \alpha \leqslant 1$

$$
\left\|x^{*}-x_{\alpha, n+1}\right\| \leqslant\left(r_{1}-t_{\alpha, n+1}\right)\left(\frac{\left\|x^{*}-x_{\alpha, n}\right\|}{r_{1}-t_{\alpha, n}}\right)^{3} .
$$

\section{MaIN ThEOREM}

We are now ready to prove the following Kantorovich-type theorem.

Theorem 3.1. Let us assume that conditions (i)-(iv) hold and also

$$
\overline{B_{\alpha}}=\overline{B\left(x_{\alpha, 1}, r_{1}-t_{\alpha, 1}\right)}=\left\{x \in X ;\left\|x-x_{\alpha, 1}\right\| \leqslant r_{1}-t_{\alpha, 1}\right\} \subseteq \Omega_{0} .
$$

Then:

(a) The sequences (2) are well-defined for $\alpha \in[0,1]$ and $n \geqslant 0$, lie in $B_{\alpha}$ (the interior of $\overline{B_{\alpha}}$ ) for $n \geqslant 1$ and converge to a solution $x^{*}$ of the equation (1).

(b) The solution is unique in $B\left(x_{0}, r_{2}\right) \cap \Omega_{0}$ if $r_{1}<r_{2}$ or in $\overline{B\left(x_{0}, r_{1}\right)} \cap \Omega_{0}$ if $r_{1}=r_{2}$.

(c) The following error estimates hold:

(c $c_{1}$ For $\alpha \in[0,1 / 2)$, let $\tau_{\alpha, n}$ and $\sigma_{\alpha, n}$ be the unique positive root and the smallest positive root of the polynomials

$$
\psi_{\alpha, n}(t)=k_{\alpha, n} t^{2}-t+\delta_{\alpha, n}
$$

and

$$
\phi_{\alpha, n}(t)=k_{\alpha, n} t^{2}+t-\delta_{\alpha, n},
$$

respectively, where

$$
k_{\alpha, n}=\frac{r_{1}-t_{\alpha, n+1}}{\left(r_{1}-t_{\alpha, n}\right)^{2}}, \quad \delta_{\alpha, n}=\left\|x_{\alpha, n+1}-x_{\alpha, n}\right\|>0 .
$$

Then we have

$$
\tau_{\alpha, n} \leqslant\left\|x^{*}-x_{\alpha, n}\right\| \leqslant \sigma_{\alpha, n} \leqslant r_{1}-t_{\alpha, n} .
$$

(c, For $\alpha \in[1 / 2,1]$, let $\tau_{\alpha, n}^{*}$ and $\sigma_{\alpha, n}^{*}$ be the smallest positive root and the unique positive root of the polynomials

$$
\phi_{\alpha, n}^{*}(t)=k_{\alpha, n}^{*} t^{3}+t-\delta_{\alpha, n},
$$

and

$$
\psi_{\alpha, n}^{*}(t)=k_{\alpha, n}^{*} t^{3}-t+\delta_{\alpha, n}
$$


respectively, where

$$
k_{\alpha, n}^{*}=\frac{r_{1}-t_{\alpha, n+1}}{\left(r_{1}-t_{\alpha, n}\right)^{3}}, \quad \delta_{\alpha, n}=\left\|x_{\alpha, n+1}-x_{\alpha, n}\right\|>0 .
$$

Then we have

$$
\sigma_{\alpha, n}^{*} \leqslant\left\|x^{*}-x_{\alpha, n}\right\| \leqslant \tau_{\alpha, n}^{*} \leqslant r_{1}-t_{\alpha, n}
$$

ProOF: (a) follows as a consequence of Lemmas 2.1, 2.6 and the condition (13). To prove (b), let $x^{* *}$ be a solution of (1) in $B\left(x_{0}, r_{2}\right) \cap \Omega_{0}$ or in $\overline{B\left(x_{0}, r_{2}\right)} \cap \Omega_{0}$. Then, replacing $x^{*}$ and $r_{1}$ by $x^{* *}$ and $r_{2}$ in Lemma 2.7 , we have for $0 \leqslant \alpha<1 / 2$,

$$
\frac{\left\|x^{* *}-x_{\alpha, n}\right\|}{r_{2}-t_{\alpha, n}} \leqslant\left(\frac{\left\|x^{* *}-x_{\alpha, n-1}\right\|}{r_{2}-t_{\alpha, n-1}}\right)^{2} \leqslant \cdots \leqslant\left(\frac{\left\|x^{* *}-x_{0}\right\|}{r_{2}}\right)^{2^{n}}
$$

In a similar way, for $1 / 2 \leqslant \alpha \leqslant 1$,

$$
\frac{\left\|x^{* *}-x_{\alpha, n}\right\|}{r_{2}-t_{\alpha, n}} \leqslant\left(\frac{\left\|x^{* *}-x_{\alpha, n-1}\right\|}{r_{2}-t_{\alpha, n-1}}\right)^{3} \leqslant \cdots \leqslant\left(\frac{\left\|x^{* *}-x_{0}\right\|}{r_{2}}\right)^{3^{n}} .
$$

So we deduce for $r_{1}<r_{2}$,

$$
\left\|x^{* *}-x_{\alpha, n}\right\| \leqslant\left(r_{2}-t_{\alpha, n}\right) \rho_{n}
$$

with

$$
\rho_{n}=\left(\frac{\left\|x^{* *}-x_{0}\right\|}{r_{2}}\right)^{2^{n}}, 0 \leqslant \alpha<1 / 2
$$

or

$$
\rho_{n}=\left(\frac{\left\|x^{* *}-x_{0}\right\|}{r_{2}}\right)^{3^{n}}, 1 / 2 \leqslant \alpha \leqslant 1
$$

For $r_{1}=r_{2}$

$$
\left\|x^{* *}-x_{\alpha, n}\right\| \leqslant r_{1}-t_{\alpha, n} .
$$

Since $\rho_{n} \rightarrow 0$ and $\left\{t_{\alpha, n}\right\}$ converges to $r_{1}$, we obtain in both cases

$$
x^{* *}=\lim _{n \rightarrow \infty} x_{\alpha, n}=x^{*}
$$

Finally, for $\alpha \in[0,1 / 2)$, we deduce from Lemma 2.7

$$
\left\|x^{*}-x_{\alpha, n}\right\|-\delta_{\alpha, n} \leqslant\left\|x^{*}-x_{\alpha, n+1}\right\| \leqslant k_{\alpha, n}\left\|x^{*}-x_{\alpha, n}\right\|^{2},
$$


and then $\phi_{\alpha, n}\left(\left\|x^{*}-x_{\alpha, n}\right\|\right) \geqslant 0$.

On the other hand,

$$
\phi_{\alpha, n}\left(r_{1}-t_{\alpha, n}\right)=k_{\alpha, n}\left(r_{1}-t_{\alpha, n}\right)^{2}-\left(r_{1}-t_{\alpha, n}\right)+\delta_{\alpha, n}=\delta_{\alpha, n}-\left(t_{\alpha, n+1}-t_{\alpha, n}\right)<0,
$$

and consequently

$$
\left\|x^{*}-x_{\alpha, n}\right\| \leqslant \sigma_{\alpha, n} \leqslant r_{1}-t_{\alpha, n} .
$$

To obtain lower bounds notice that, by using Lemma 2.7 again,

$$
\delta_{\alpha, n}-\left\|x^{*}-x_{\alpha, n}\right\| \leqslant\left\|x^{*}-x_{\alpha, n+1}\right\| \leqslant k_{\alpha, n}\left\|x^{*}-x_{\alpha, n}\right\|^{2},
$$

that is, $\psi_{\alpha, n}\left(\left\|x^{*}-x_{\alpha, n}\right\|\right) \geqslant 0$. Consequently,

$$
\left\|x^{*}-x_{\alpha, n}\right\| \geqslant \tau_{\alpha, n} .
$$

For $\alpha \in[1 / 2,1]$, we obtain from Lemma 2.7

$$
\left\|x^{*}-x_{\alpha, n}\right\|-\delta_{\alpha, n} \leqslant\left\|x^{*}-x_{\alpha, n+1}\right\| \leqslant k_{\alpha, n}\left\|x^{*}-x_{\alpha, n}\right\|^{3} .
$$

and

$$
\delta_{\alpha, n}-\left\|x^{*}-x_{\alpha, n}\right\| \leqslant\left\|x^{*}-x_{\alpha, n+1}\right\| \leqslant k_{\alpha, n}\left\|x^{*}-x_{\alpha, n}\right\|^{3},
$$

and the result also holds.

Note. The condition (13) can be replaced by $\overline{B\left(x_{0}, r_{1}\right)} \subseteq \Omega_{0}$. (Notice that $\overline{B_{\alpha}}$ $\subseteq \overline{B\left(x_{0}, r_{1}\right)}$ for $\alpha \in[0,1]$.)

Corollary 3.2. Under the previous assumptions, we have

$$
0.8 \delta_{\alpha, n}<(-2+\sqrt{8}) \delta_{\alpha, n} \leqslant\left\|x^{*}-x_{\alpha, n}\right\| \leqslant 2 \delta_{\alpha, n}, \quad \alpha \in[0,1 / 2),
$$

and

$$
0.89 \delta_{\alpha, n} \leqslant\left\|x^{*}-x_{\alpha, n}\right\| \leqslant 1.5 \delta_{\alpha, n}, \quad \alpha \in[1 / 2,1] .
$$

Proof: Let $\alpha \in[0,1 / 2)$. The polynomial $\phi_{\alpha, n}$ has a minimum when $t=1 /\left(2 k_{\alpha, n}\right)$. Also

$$
\phi_{\alpha, n}\left(\frac{1}{2 k_{\alpha, n}}\right)=\delta_{\alpha, n}-\frac{1}{4 k_{\alpha, n}}<0 .
$$

Consequently $k_{\alpha, n} \delta_{\alpha, n}<1 / 4$. Since $\phi_{\alpha, n}\left(2 \delta_{\alpha, n}\right)<0$, we deduce $\sigma_{\alpha, n}<2 \delta_{\alpha, n}$.

On the other hand, let

$$
\tilde{\psi}_{\alpha, n}(t)=\frac{1}{4 \delta_{\alpha, n}} t^{2}+t-\delta_{\alpha, n} .
$$

As $\psi_{\alpha, n}(t)<\widetilde{\psi}_{\alpha, n}(t)$ for $t>0$, and $\widetilde{\psi}_{\alpha, n}\left((-2+\sqrt{8}) \delta_{\alpha, n}\right)=0$, we obtain $(-2+\sqrt{8}) \delta_{\alpha, n}<\tau_{\alpha, n}$.

For $\alpha \in[1 / 2,1]$ the result follows in a similar way (see [20] for details). 
Table 1

\begin{tabular}{|c|c|c|c|}
\hline$n$ & $t_{0, n}$ & $t_{1 / 2, n}$ & $t_{1, n}$ \\
\hline 0 & 0.000000000000000 & 0.000000000000000 & 0.000000000000000 \\
1 & 8.148148148148148 & 8.571428571428571 & 9.333333333333333 \\
2 & 9.936934743362793 & 9.980430528375734 & 9.999847409781033 \\
3 & 9.999995122930333 & 9.999999925494193 & 10.000000000000000 \\
4 & 10.000000000000000 & 10.000000000000000 & 10.000000000000000 \\
5 & 10.000000000000000 & 10.000000000000000 & 10.000000000000000 \\
\hline
\end{tabular}

\section{EXAMPLES}

EXAmple 1. First we consider the real polynomial equation

$$
p(t) \equiv(t-10)(t-20)=t^{2}-30 t+200=0 .
$$

In Table 1 we compare the sequences $\left\{t_{0, n}\right\}$ (Chebyshev's method), $\left\{t_{1 / 2, n}\right\}$ (Halley's method) and $\left\{t_{1, n}\right\}$ (convex acceleration of Newton's method), starting from the same point $t_{0,0}=t_{1 / 2,0}=t_{1,0}=0$.

EXAMPLE 2. Now we consider the system of equations $F(x, y)=0$, where

$$
F(x, y)=\left(x^{2}-y-2, y^{3}-x^{2}+y+1\right) \text {. }
$$

Starting at $\left(x_{0,0}, y_{0,0}\right)=\left(x_{1 / 2,0}, y_{1 / 2,0}\right)=\left(x_{1,0}, y_{1,0}\right)=(6,3)$ we obtain the sequences given in Tables 2-4.

In the previous examples we have analysed the velocity of convergence of different sequences of the family (2), attaining the best results for $\alpha=1$. In Theorem 3.1 we have only studied the sequences $\left\{x_{\alpha, n}\right\}$ for $\alpha \in[0,1]$. However, it is possible to obtain convergent sequences for $\alpha>1$, and the convergence could be even faster, as happens in the real case. It is not difficult to see from (7) that $G^{\prime \prime \prime}\left(r_{1}\right)=0$ for

$$
\alpha=1-\frac{L_{p^{\prime}}\left(r_{1}\right)}{3} \geqslant 1 \text {. }
$$

Then, the order of convergence increases for this value. For instance, when $p^{\prime \prime}(t)$ is constant, we obtain a fourth-order method for $\alpha=1$, as was remarked in [12]. This result was extended to Banach spaces in the same paper.

The following example suggest new approaches to the solution of a integral equation (see also $[4,5,9]$ ). 
Table 2. Chebyshev's method

\begin{tabular}{|c|c|c|}
\hline$n$ & $x_{0, n}$ & $y_{0, n}$ \\
\hline 0 & 6.000000000000000000 & 3.000000000000000000 \\
1 & 2.719439840217446527 & 1.727937814357567444 \\
2 & 1.830721648025601499 & 1.138820258896789643 \\
3 & 1.733249320860063453 & 1.003038870277247239 \\
4 & 1.732050823057711034 & 1.000000046348271498 \\
5 & 1.732050807568877294 & 1.000000000000000000 \\
6 & 1.732050807568877294 & 1.000000000000000000 \\
\hline
\end{tabular}

Table 3. Halley's method

\begin{tabular}{|c|c|c|}
\hline$n$ & $x_{1 / 2, n}$ & $y_{1 / 2, n}$ \\
\hline 0 & 6.000000000000000000 & 3.000000000000000000 \\
1 & 2.527771600300525920 & 1.581818181818181818 \\
2 & 1.774166519412615274 & 1.057032561548124306 \\
3 & 1.732099394264064235 & 1.000113501450570644 \\
4 & 1.732050807569220348 & 1.000000000000974628 \\
5 & 1.732050807568877294 & 1.000000000000000000 \\
6 & 1.732050807568877294 & 1.000000000000000000 \\
\hline
\end{tabular}

Table 4. Convex acceleration of Newton's method

\begin{tabular}{|c|c|c|}
\hline$n$ & $x_{1, n}$ & $y_{1, n}$ \\
\hline 0 & 6.000000000000000000 & 3.000000000000000000 \\
1 & 2.195930445526441461 & 1.173690932311621967 \\
2 & 1.726757444904059338 & 0.998981852656109923 \\
3 & 1.732050203990691682 & 1.000000000352888045 \\
4 & 1.732050807568877294 & 1.000000000000000000 \\
5 & 1.732050807568877294 & 1.000000000000000000 \\
\hline
\end{tabular}


EXAmple 3. In the space $X=C[0,1]$ of all continous functions on the interval $[0,1]$ with the norm

$$
\|x\|=\max _{s \in[0,1]}|x(s)|,
$$

we consider the equation $F(x)=0$, where

$$
F(x)(s)=x(s)-s+\frac{1}{2} \int_{0}^{1} s \cos (x(t)) d t, \quad x \in C[0,1], s \in[0,1] .
$$

With the notation of Theorem 3.1 and for $x_{0}=x_{0}(s)=s$, we use the definition of the first and second Fréchet derivatives of the operator $F$ to obtain

$$
a=b=\frac{\sin 1}{2-\sin 1+\cos 1}, \quad k=\frac{1}{2-\sin 1+\cos 1} .
$$

So the polynomial (3) is

$$
p(t)=\frac{1}{6(2-\sin 1+\cos 1)}\left[t^{3}+3(\sin 1) t^{2}-6(2-\sin 1+\cos 1) t+6 \sin 1\right] .
$$

The positive roots of $p$ are

$$
r_{1}=0.6095694860276291, \quad r_{2}=1.70990829134757 .
$$

Then, we have that $F(x)=0$ has a root in $\overline{B\left(x_{0}, r_{1}\right)}$. Besides, this is the unique root in $B\left(x_{0}, r_{2}\right)$. Some error bounds

$$
\left\|x^{*}-x_{\alpha, n}\right\| \leqslant r_{1}-t_{\alpha, n}
$$

are shown in Table 5.

Table 5. Error bounds

\begin{tabular}{|c|c|c|c|}
\hline$n$ & $r_{1}-t_{0, n}$ & $r_{1}-t_{1 / 2, n}$ & $r_{1}-t_{1, n}$ \\
\hline 0 & 0.6095694860276291 & 0.6095694860276291 & 0.6095694860276291 \\
1 & 0.0534834955243040 & 0.0495130055348865 & 0.0349873303274992 \\
2 & 0.0001520166774545 & 0.0000984825547302 & 0.0000560164474543 \\
3 & 0.0000000000042804 & 0.0000000000009129 & 0.0000000000001218 \\
4 & 0.0000000000000000 & 0.0000000000000000 & 0.0000000000000000 \\
\hline
\end{tabular}


Notice that the best error bounds are attained when $\alpha=1$ (convex acceleration of Newton's method). For this same equation, and using Halley's method, Döring [9] obtained the bound

$$
\left\|x^{*}-x_{1 / 2,2}\right\| \leqslant 0.000825 \text {. }
$$

Later, Candela and Marquina, $[4,5]$, gave the bounds

$$
\left\|x^{*}-x_{0,2}\right\| \leqslant 0.00037022683427694
$$

and

$$
\left\|x^{*}-x_{1 / 2,2}\right\| \leqslant 0.00014987029635502
$$

for Chebyshev's and Halley's methods respectively. Observe that the bounds given in Table 5 really improve the previous ones.

\section{REFERENCES}

[1] G. Alefeld, 'On the convergence of Halley's method', Amer. Math. Monthly 88 (1981), 530-536.

[2] M. Altman, 'Concerning the method of tangent hyperbolas for operator equations', Bull. Acad. Pol. Sci., Ser. Sci. Math., Ast. et Phys. 9 (1961), 633-637.

[3] I.K. Argyros and D. Chen, 'Results on the Chebyshev method in Banach spaces', Proyecciones 12 (1993), 119-128.

[4] V. Candela and A. Marquina, 'Recurrence relations for rational cubic methods I: The Halley method', Computing 44 (1990), 169-184.

[5] V. Candela and A. Marquina, 'Recurrence relations for rational cubic methods II: The Chebyshev method', Computing 45 (1990), 355-367.

[6] D. Chen, 'Ostrowski-Kantorovich theorem and S-order of convergence of Halley method in Banach spaces', Comment. Math. Univ. Carolin. 34 (1993), 153-163.

[7] D. Chen, I.K. Argyros and Q.S. Qian, 'A note on the Halley method in Banach spaces', Appl. Math. Comput. Sci. 58 (1993), 215-224.

[8] D. Chen, I.K. Argyros and Q.S. Qian, 'A local convergence theorem for the Super-Halley method in a Banach space', Appl. Math. Lett. 7 (1994), 49-52.

[9] B. Döring, 'Einige Sätze über das verfahren der tangierenden hyperbeln in Banach-Räumen', Aplikace Mat. 15 (1970), 418-464.

[10] W. Gander, 'On Halley's iteration method', Amer. Math. Monthly 92 (1985), 131-134.

[11] J.M. Gutiérrez, M.A. Hernández and M.A. Salanova, 'Accesibility of solutions by Newton's method', Intern. J. Computer Math. 57 (1995), 239-247.

[12] J.M. Gutiérrez, Newton's method in Banach spaces, Ph.D. Thesis (University of La Rioja, Logroño, 1995).

[13] M.A. Hernández, 'A note on Halley's method', Numer. Math. 58 (1991), 273-276.

[14] M.A. Hernández, 'Newton-Raphson's method and convexity', Zb. Rad. Prirod.-Mat. Fak. Ser. Mat. 22 (1993), 159-166. 
[15] M.A. Hernández and M.A. Salanova, 'A family of Chebyshev-Halley type methods', Intern. J. Computer Math. 47 (1993), 59-63.

[16] L.V. Kantorovich and G.P. Akilov, Functional analysis (Pergamon Press, Oxford, 1982).

[17] A.M. Ostrowski, Solution of equations in Euclidean and Banach spaces (Academic Press, New York, 1943).

[18] L.B. Rall, Computational solution of nonlinear operator equations (Robert E. Krieger Publishing Company, Inc., New York, 1979).

[19] W.C. Rheinboldt, 'A unified convergence theory for a class of iterative process', SIAM J. Numer. Anal. 5 (1968), 42-63.

[20] T. Yamamoto, 'On the method of tangent hyperbolas in Banach spaces', J. Comput. Appl. Math. 21 (1988), 75-86.

Dpt Matemáticas y Computación

Universidad de La Rioja

26004 Logroño

Spain

e-mail: jmguti@siur.unirioja.es

mahernan@siur.unirioja.es 